\title{
Philosophiques
}

\section{Index du volume 34 (2007)}

Volume 34, numéro 2, automne 2007

URI : https://id.erudit.org/iderudit/017434ar

DOI : https://doi.org/10.7202/017434ar

Aller au sommaire du numéro

Éditeur(s)

Société de philosophie du Québec

ISSN

0316-2923 (imprimé)

1492-1391 (numérique)

Découvrir la revue

Citer ce document

(2007). Index du volume 34 (2007). Philosophiques, 34(2), 435-436.

https://doi.org/10.7202/017434ar

Ce document est protégé par la loi sur le droit d'auteur. L'utilisation des services d'Érudit (y compris la reproduction) est assujettie à sa politique d'utilisation que vous pouvez consulter en ligne.

https://apropos.erudit.org/fr/usagers/politique-dutilisation/
Cet article est diffusé et préservé par Érudit.

Érudit est un consortium interuniversitaire sans but lucratif composé de l'Université de Montréal, l'Université Laval et l'Université du Québec à Montréal. Il a pour mission la promotion et la valorisation de la recherche. https://www.erudit.org/fr/ 


\section{Index du volume 34 (2007)}

\section{Articles}

BRISART, ROBERT, Les premières articulations du fonctionnement intentionnel: le projet d'un Raumbuch chez Husserl entre 1892 et 1894

Chung, Ryoa, Domination, vulnérabilité et inégalité d'accès aux soins de santé

Couture, Jocelyne et Nielsen, KaI, Introduction: Cosmopolitisme et particularisme

GAGNé, LEARRY, La modélisation des comportements non conséquentialistes en théorie du choix rationnel

Galibert, Pablo, La justice globale, le multiculturalisme et les revendications des immigrants

Lima, Maria Herrera, Prendre les autres au sérieux: sur le débat cosmopolitique-particularisme

MACLURE, JOCELYN, La reconnaissance engage-t-elle à l'essentialisme?

Moellendorf, Darrel, La justice et les associations

SMith, Justin E. H., La génération spontanée et le problème de la reproduction des espèces avant et après Descartes

TAN, KOK-CHOR, Nationalisme libéral et internationalisme égalitaire

Vanderveken, Daniel, Principes de pragmatique formelle du discours

VILMER, JeAn-BAPTISTE, La prudence de Descartes face à la question de l'infini en mathématiques

WENAR, LEIF, L'individu, l'État et les droits de base

Wolfe, Charles T., Le rêve matérialiste, ou "Faire par la pensée ce que la matière fait parfois"

\section{Disputationes}

Bishop, Michael, Notice critique : Reconstructing Reason and Representation - Murray Clarke

Clarke, Murray, Précis de Reconstructing Reason and Representation

Clarke, Murray, Réponses à mes critiques

FAUCHER, LuC, L'intentionnalité, naturellement!

FAucher, Luc, Faraway, so close?

Hudson, Robert, Améliorer les théories de la fiabilité

Jacob, Pierre, Précis de L'Intentionnalité, problèmes de philosophie de l'esprit

JACOB, Pierre, Réponses à mes critiques

VALLÉE, RiCHARD, Intentionnalité et référence directe 


\section{Philosophiques / Automne 2007}

\section{Études critiques}

Hacker, P.M.R. and Bennett, M. R. Philosophical Foundations of Neuroscience (Pierre Poirier et Nicolas Payette)

Arpaly, N., Unprincipled Virtue. An Inquiry into Moral Agency

(François Schroeter)

\section{Comptes rendus}

Beaulieu, Alain, (dir.), Gilles Deleuze, héritage philosophique

(Charles Bolduc)

Filion, Jean-François, Dialectique et matière. La conceptualité inconsciente des processus inorganiques dans la Philosophie de la nature (1830) de Hegel (Danic Parenteau)

Gnassounou, Bruno, et Kistler, Max, Causes, pouvoirs, dispositions en philosophie. Le retour des vertus dormitives (Aurélien Robert)

Khushf, George, (dir.), Handbook of bioethics: Taking stock of the field from a philosophical perspective (Soumaya Mestiri)

RESCHER, Nicolas, Essais sur les fondements de l'ontologie du procès (Frédéric Tremblay)

Rescher, Nicolas, Metaphysics: The Key Issues From A Realistic Perspective (Frédéric Tremblay)

Seidengart, Jean, Dieu, l'univers et la sphère infinie. Penser l'infinité cosmique à l'aube de la science classique (Yvon Gauthier)

Seymour, Michel, L'institution du langage (Pascal Engel)

Stelzner, Werner und Kreiser, Lothar, Traditionelle und nichtklassische Logik (Guillaume Fréchette)

Zarka, Yves Charles, Un détail nazi dans la pensée de Carl Schmitt (Luc Vigneault)

Zеimbeкis, John. Qu'est-ce qu'un jugement esthétique? (Mélissa Thériault) 